\title{
LinA como ferramenta auxiliar no processo de ensino-aprendizagem de Álgebra Linear
}

\author{
Cleriston Dantas ${ }^{1}$, Vaston G. da Costa $^{2}$, Salviano Sandoval Ludgerio ${ }^{3}$ \\ ${ }^{1}$ DevUP Soluções
}

Trv rua 8, n 23, Res. dos Lucas - Catalao, GO - Brazil

${ }^{2}$ Instituto de Matemática e Tecnologia - Universidade Federal de Goiás (UFG)

Av. Dr. Lamartine P. de Avelar, 1120 - Catalao, GO - Brazil

${ }^{3}$ Instituto de Biotecnologia - Universidade Federal de Goiás (UFG)

Av. Dr. Lamartine P. de Avelar, 1120 - Catalao, GO - Brazil

cleriston@devupsolucoes.com.br, vaston@ufg.br, salviano.lfg@gmail.com

\begin{abstract}
Resumo. A disciplina de álgebra linear para os cursos de exatas é obrigatória, notadamente, pela grande aplicação dos conceitos em outras disciplinas dos cursos, inclusive naquelas específicas da profissão elou de pós-graduação. Mesmo com o grande avanço de sistemas voltados para o ensino de conceitos matemáticos, a sua efetiva aplicação em sala de aula esbarra na falta de estrutura física necessária para abarcar o quantitativo de alunos que geralmente cursam tal disciplina. Visando levar a tecnologia às salas de aulas da disciplina de álgebra linear e àquelas que empregam o ferramental de álgebra linear, que se propôs a criação de um aplicativo para dispositivos móveis com sistema operacional Android ${ }^{\circledR}$. O protótipo foi concebido, desenvolvido e aplicado em uma turma de Álgebra Linear do curso de ciência da Computação da Universidade Federal de Goiás.
\end{abstract}

\section{Cenário de Uso}

A disciplina de álgebra é notadamente importante para os cursos superiores na área de exatas, seja para suas aplicações em disciplinas futuras da grade curricular do curso, seja para aplicações posteriores à graduação.

Há contudo um grande problema de assimilação dos conteúdos de álgebra por parte dos alunos [Sierpinska 2000, Dorier et al. 2000]. E as explicações para tal problema são, dentre outras, a letargia presente na atualização de métodos de ensino das disciplinas de cunho matemático [Harel 2000] e a falha na formação matemática dos alunos que ingressam nas universidades [Robert 2000].

O uso de tecnologia como auxiliar no processo ensino-aprendizagem é algo que se propõe há muito [Lévy 1996, Figueiredo et al. 2015]. Em particular, para o ensino de conceitos matemáticos, pode-se destacar o amplo desenvolvimento de softwares que podem ser empregados no processo ensino-aprendizagem. Dentre tais, pode-se destacar Matlab ${ }^{\circledR}$, Maple ${ }^{\mathrm{R}}$, Poly ${ }^{\mathrm{R}}$, Super Logo ${ }^{\mathrm{R}}$ e Geogebra ${ }^{\mathrm{R}}$. Exceto pelo último, todos são sistemas computacionais de instalação em computadores pessoais e alguns, como Matlab ${ }^{\circledR}$, podem ser empregados em tarefas muitos complexas que demandam grande quantidade de processamento e de muito conhecimento por parte dos utilizadores. 
Como afirma Prensky em [Prensky 2001], os jovens ingressantes em cursos superiores são os denominados "nativos digitais" no sentido de que já nasceram imersos num mundo tecnológico e fazem uso constante da tecnologia em seus afazeres diários. A resistencia, portanto, em se empregar softwares matemáticos como apoio ao processo de ensino-aprendizagem parte, muitas das vezes, dos professores, denominados de "imigrantes digitais". Mesmo havendo vários relatos de casos de sucesso que atestam ganho [Harel 2000, Fonseca 2014].

Os educadores que pretendem empregar a tecnologia em sala de aula esbarram em problemas de infraestrutura física e tecnológica inadequada, na pouca prática para se explorar as potencialidades dos recursos disponíveis, falta de profissionais para a gestão e manutenção dos laboratórios e na facilidade de dispersão do aluno em laboratórios [Figueiredo et al. 2015].

Uma alternativa que se apresenta é a de fazer uso de uma tecnologia simples, que não demande uma estrutura física suplementar e nem manutenção contínua. Tendo por simplicidade tanto de utilização quanto de aquisição.

Com esta perspectiva em mente que se desenvolveu o sistema para dispositivos móveis que auxilia o professor e os alunos de álgebra linear na compreensão de conteúdos básicos da disciplina - escalonamento, determinantes e inversão de matrizes - e que se comportasse como uma calculadora. O sistema foi desenvolvido apenas para dispositivos com sistema operacional Android ${ }^{\mathrm{R}}$ e nova versão com mais funcionalidades e para novos sistemas está em fase de implementação.

O sistema, denominado LinA, foi produzido em conjunto com alunos da disciplina de álgebra linear ministrada ao curso de ciência da computação e passou por testes de usabilidade com os alunos e o professor envolvidos e com professores do departamento de matemática.

O sistema se mostrou simples, barato e prático para ser empregados por alunos em sala de aula, sem necessidade de espaço fisico específico, e durante a prática de exercícios. Também é muito útil quando os conceitos de álgebra são necessários para se resolver exercícios de outras disciplinas como, por exemplo, cálculo em várias variáveis e equações diferenciais.

\section{Desenvolvimento}

A metodologia empregada para o desenvolvimento levou em consideração aspectos pedagógicos relativos à motivação e ao aprendizado do aluno participante da disciplina de álgebra linear, visando apresentar-lhe o universo de desenvolvimento de sistemas para aplicativos móveis bem como o processo algoritmo empregado na disciplina de álgebra linear. Em especial, fases de estudo teórico foram intercaladas com fases de implementação e de utilização do aplicativo em desenvolvimento.

Foram utilizados conceitos da metodologia RUP (Rational Unified Process) e princípios dos chamados Métodos Ágeis de Desenvolvimento de Software [Cockburn 2002] que prevêem a construção de software de forma incremental, iterativa e adaptativa. Esta escolha se deve à necessidade de utilizar um processo ágil de desenvolvimento, que, além de ser fácil gerenciado, também é razoavelmente bem documentado. Sempre que possível, o desenvolvimento incremental do software se baseou em técnicas 
V Congresso Brasileiro de Informática na Educação (CBIE 2016)

Anais dos Workshops do V Congresso Brasileiro de Informática na Educação (CBIE 2016)

de refatoração [Fowler et al. 1999]. Aspectos estruturais e comportamentais do software foram representados através de diagramas UML e, sempre que possível, foram adotados padrões arquiteturais e de projeto consagrados [Gamma 1995].

Para o desenvolvimento do sistema proposto, foram utilizadas diversas ferramentas, como o gerador automático de documentação Javadoc e o arcabouço para realização de testes de unidade JUnit. Para garantir uma gerência eficiente das versões do software gerado ao longo do processo de desenvolvimento e permitir o desenvolvimento colaborativo, foi utilizado o software de controle de versões GIT. Adotou-se um estilo de programação adequado à linguagem Java na implementação dos componentes da plataforma JDK-SDKAndroid.

Por conta da proposta de desenvolvimento, testes de usabilidade foram realizadas ao término de cada novo módulo, mais uma justificativa para o emprego de métodos ágeis para o desenvolvimento.

\section{Apresentação do software}

O LinA possui uma interface inicial conforme figura 1, em que o usuário pode escolher entre escalonar, verificar dependência linear ou inverter matriz. Nesta tela também é informado o formato de entrada de dados que representa a matriz. No caso, separa-se os elementos de linha por vírgulas e as colunas são separados por ponto-virgula.

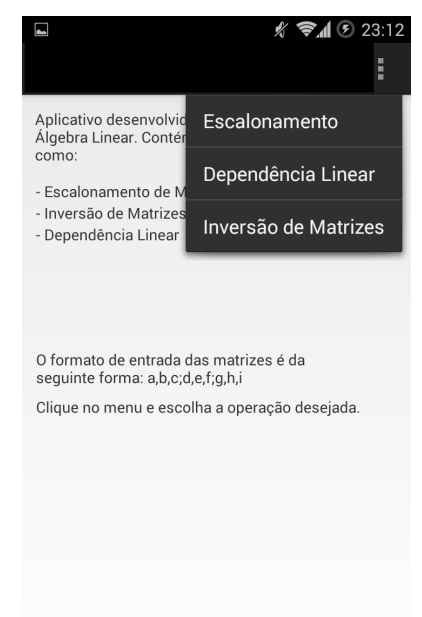

Figura 1. Tela de abertura

Se o usuários escolher por realizar escalonamento, é apresentada a tela conforme figura 2(a).

Após inserir os valores da matriz, na linha superior, é possível ao usuário verificar como a matriz se apresenta bastando, para isto, clicar no botão "Ver Matriz". Ao clicar neste botão será exibida a matriz, conforme figura 2(b). Se o usuário clicar em "Escalonar", a matriz será escalonada e o determinantes será apresentado 2(c)

De fato, o melhor uso para o aplicativo é em servir como calculadora em que o usuário possa aprender com os passos de execução. LinA apresenta esta possibilidade 
V Congresso Brasileiro de Informática na Educação (CBIE 2016)

Anais dos Workshops do V Congresso Brasileiro de Informática na Educação (CBIE 2016)

ao permitir que o usuário realize operações elementares com os elementos da matriz de entrada: Multiplicar uma linha por um valor constante 3(c) e soma linhas 3(a) e apresenta a nova matriz gerada após cada operação 3(d) e 3(b).

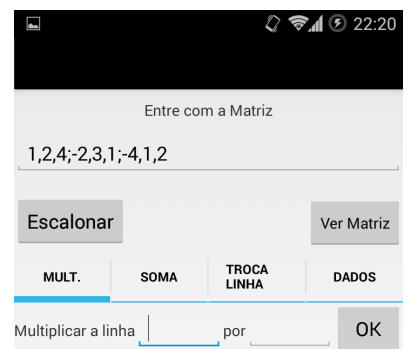

(a) Tela inicial
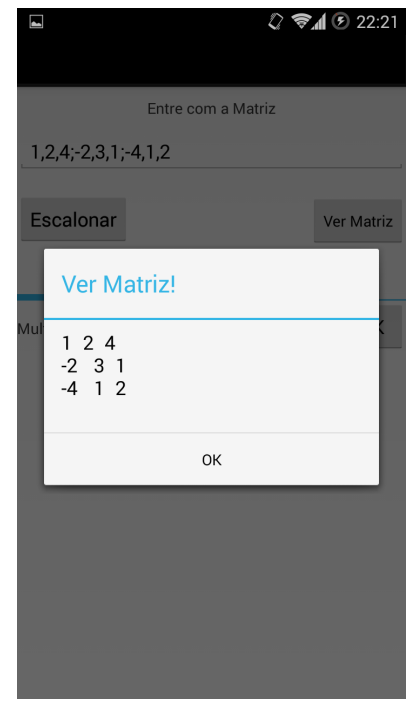

(b) Ver Matriz

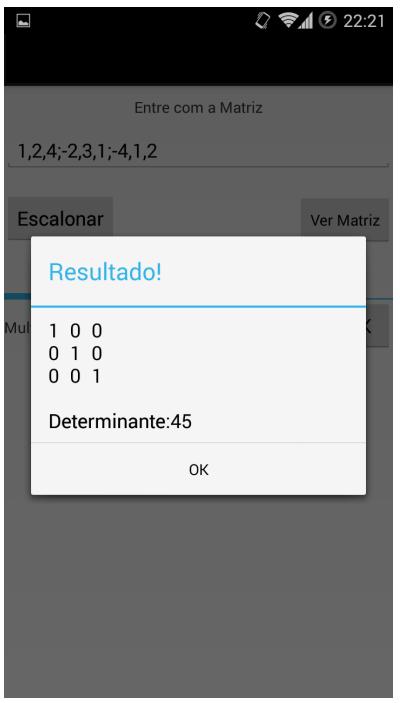

(c) Escalodada

Figura 2. Escalonamento Automático

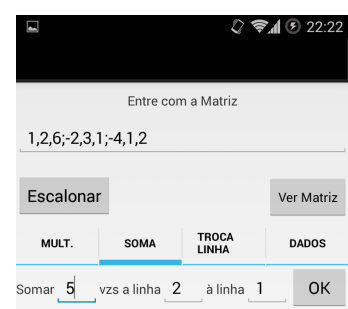

(a) Somando linhas

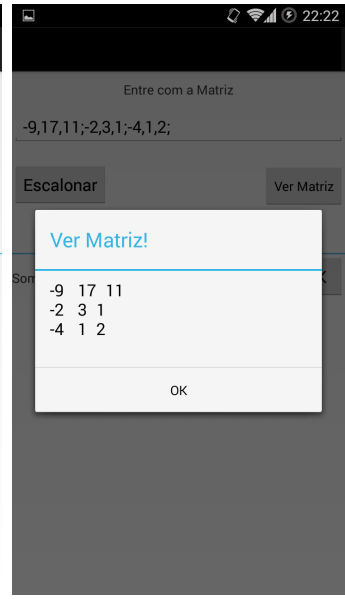

(b) Ver soma de linhas

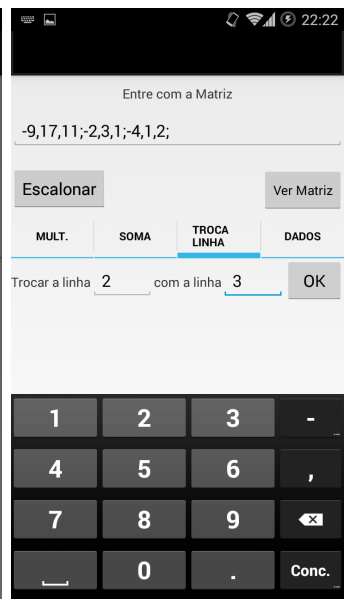

(c) Multiplicando por constante

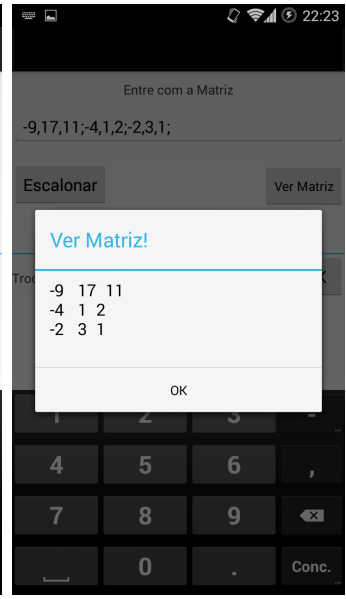

(d) Ver multiplicação

Figura 3. Escalonamento

Na opção Dependência Linear, no menu de entrada, o usuário pode empregar o sistema para verificar se vetores são linearmente dependentes ou linearmente independentes. Bastando que o usuário enter com vetores em forma de matriz, na linha superior do aplicativo, clicando em "Ver matriz"(figura 4(a)) é possível verificar a matriz inserida.

Para visualizar o resultado, sem cálculo intermediários, basta clicar em "Verificar". Se o sistema for linearmente independente surgirá a resposta "Sistema linearmente 
V Congresso Brasileiro de Informática na Educação (CBIE 2016)

Anais dos Workshops do V Congresso Brasileiro de Informática na Educação (CBIE 2016)

independente". Caso contrário, surgirá a mensagem "Ops, ainda não está na forma reduzida"(figura 4(b)).

Contudo, para efeitos de aprendizado, o interface de Dependência Linear permite que o usuários verifique passo-a-passo via operações elementares se os vetores são, ou não, linearmente dependentes.

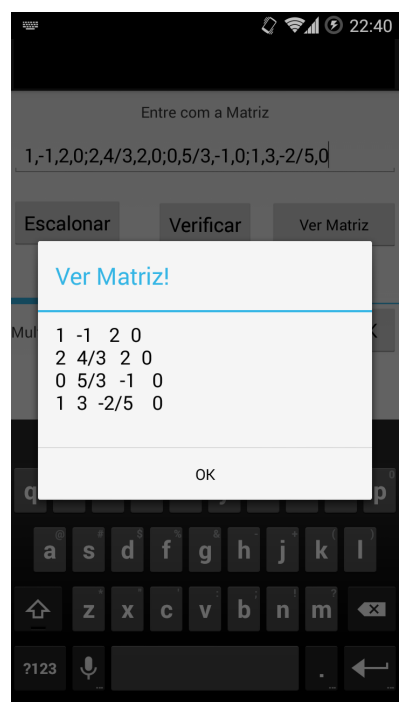

(a) Dependencia 1

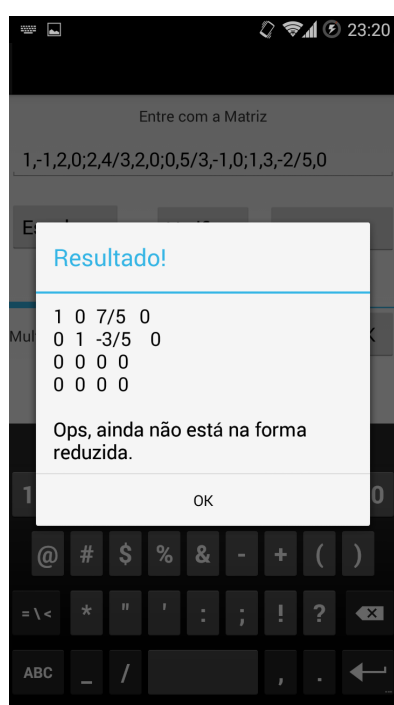

(b) Dependencia 2

Figura 4. Dependencia Linear

A terceira funcionalidade do aplicativo permite o usuário determinar a matriz inversa. Tanto diretamente, clicando "Inverter", quanto passo-a-passo, inserindo valores por operações elementares, assim como nas demais funcionalidades. Na figura 5 é apresentado o resultado de inversão da matriz $\left[\begin{array}{ccc}1 & 2 & 4 \\ -2 & 3 & 1 \\ -4 & 1 & 2\end{array}\right]$.

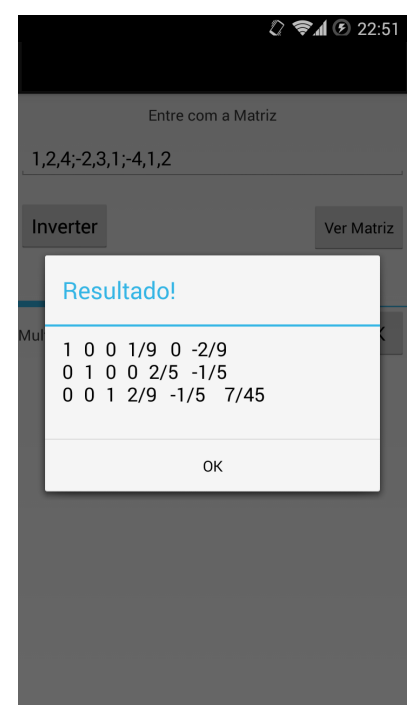

Figura 5. Inversa 
V Congresso Brasileiro de Informática na Educação (CBIE 2016)

Anais dos Workshops do V Congresso Brasileiro de Informática na Educação (CBIE 2016)

O sistema pode ser encontrado em http://vivacatalao.com.br/ index.php?app e a instalação segue o padrão de todos os aplicativos da Google Play ${ }^{R}$.

O video que demonstra as funcionalidades está disponível em http:// vivacatalao.com.br/index.php?video.

O software, ainda protótipo, está sob licença Creative Commons BY-NC-SA.

\section{Considerações finais}

O LinA é um sistema desenvolvidos para dispositvos móveis com sistema operacional Android ${ }^{\circledR}$ e que pode ser utilizado como uma calculadora de álgebra linear .

É de fácil emprego por professores e de fácil utilização pelos alunos "nativos tecnológicos" vez que não necessita de estrutura física de laboratório de ensino para ser utilizado nem, tampouco, de conhecimento aprofundado sobre seu uso.

O sistema passou por testes de unidade e testes de usabilidade e conta com uma boa documentação.

A próxima etapa de evolução consiste em estender LinA para outros sistemas operacionais de dispositivos móveis e criar uma versão web.

\section{Referências}

Cockburn, A. (2002). Agile software development. Agile software development series. Addison-Wesley.

Dorier, J.-L., Robert, A., Robinet, J., and Rogalsiu, M. (2000). The Obstacle of Formalism in Linear Algebra, pages 85-124. Springer Netherlands, Dordrecht.

Figueiredo, G. L. R., Nobre, I. A. M., and Passos, M. L. S. (2015). Tecnologias computacionais na educação: Desafios na prática docente.

Fonseca, J. R. S. (2014). Reduzir as atitudes negativas em relação à aprendizagem de Matemática e aumentar o desempenho dos alunos através da metodologia CAL. Revista Brasileira de Informática na Educação, 22(1):121-131.

Fowler, M., Beck, K., Brant, J., Opdyke, W., and Roberts, D. (1999). Refactoring: Improving the Design of Existing Code. Addison-Wesley Professional.

Gamma, E. (1995). Design patterns: elements of reusable object-oriented software. Addison-Wesley professional computing series. Addison-Wesley.

Harel, G. (2000). Three Principles of Learning and Teaching Mathematics, pages 177189. Springer Netherlands, Dordrecht.

Lévy, P. (1996). As tecnologias da inteligência: o futuro do pensamento na era da informática. 34 Literatura S/C Ltda, São Paulo, 3 edition.

Prensky, M. (2001). Digital natives, digital immigrants part 1. On the Horizon, 9(5):1-6.

Robert, A. (2000). Level of Conceptualization and Secondary School Math Education, pages 125-131. Springer Netherlands, Dordrecht.

Sierpinska, A. (2000). On Some Aspects of Students' Thinking in Linear Algebra, pages 209-246. Springer Netherlands, Dordrecht. 